\title{
IgA deficiency: a risk factor for food allergy-related atopic dermatitis in infants and young children
}

\author{
Aleksandra Szczawińska-Popłonyk, Paulina Komasińska, Anna Bręborowicz \\ Department of Pediatric Pneumonology, Allergology and Clinical Immunology, Poznan University of Medical Sciences, Poznan, Poland
}

Adv Dermatol Allergol 2016; XXXIII (5): 369-374

DOI: 10.5114/ada.2016.62844

\begin{abstract}
Introduction: The impaired immunosurveillance and dysfunctional antigen compartmentalization in primary immunodeficiencies may predispose affected children to allergic sensitization.

Aim: The growing problem of allergic reactions to foods and the increase in non-lgE-mediated gastrointestinal disorders prompted us to investigate the impact of hypogammaglobulinemia on the prevalence and clinical phenotypes of food allergy.

Material and methods: We performed a retrospective analysis of medical records of 78 infants and young children with deficiencies of one or more immunoglobulin isotypes and of 132 age-matched patients with normal immunoglobulin production in terms of prevalence and clinical manifestation of food allergy, atopic sensitization and physical development.

Results: The symptoms of allergy to foods were demonstrated with a comparable frequency in children with hypogammaglobulinemia and with normal immunoglobulin levels ( $51 \%$ vs. $48 \%$ ). The most prominent clinical phenotype of children with hypogammaglobulinemia was atopic dermatitis, whereas in children with normal immunoglobulin production, gastrointestinal symptomatology predominated. Uniquely, IgA deficiency showed a statistically significant $(p<0.05)$ correlation with food allergy and with cutaneous symptomatology. A striking predominance of non-lgE-mediated food allergy was demonstrated in both groups of the children studied, with elevated serum total IgE levels seen more commonly in children with normogammaglobulinemia than in those with hypogammaglobulinemia.

Conclusions: The rate of non-IgE mediated allergic sensitization to foods in the population of infants and young children is high and clinically relevant. A selective IgA deficiency should alert physicians to assess affected children with respect to diverse phenotypes of food allergy.
\end{abstract}

Key words: antibody deficiency, allergy, atopic dermatitis, children.

\section{Introduction}

Primary antibody deficiencies (PADs) are the most prevalent group of congenital defects of the immune system accounting for more than $50 \%$ of all immunodeficiency disorders in the pediatric population [1]. They comprise a number of genetically, clinically and pathogenetically heterogeneous diseases, with the manifestation ranging from often a mild or even asymptomatic immunoglobulin A (IgA) deficiency or IgG subclass deficiency, to severe agammaglobulinemias, associated with serious infections and multiorgan long-term complications [2]. As impaired ability to mount an effective antigen-specific antibody immune response is the major feature of PADs, increased susceptibility to infections is a common clinical manifestation of hypogammaglobulinemia in children. However, the phenotypic expression resulting from disturbed immunosurveillance in primary immunodeficiencies may be diverse and the allergy, along with autoimmunity and lymphoproliferation, significantly contributes to the heterogeneity of the clini-

Address for correspondence: Assoc. Prof. Aleksandra Szczawińska-Popłonyk MD, PhD, Department of Pediatric Pneumonology, Allergology and Clinical Immunology, Poznan University of Medical Sciences, 27/33 Szpitalna St, 60-572 Poznan, Poland, phone: +48 6184801 11, e-mail: ola@malwa.com.pl Received: 24.08.2015, accepted: 22.10.2015. 
cal manifestation. Besides well-defined disease entities with a predominant intrinsic B lymph cell defect such as X-linked agammaglobulinemia due to Bruton's tyrosine kinase (Btk) deficiency, a concomitant T lymph cell dysfunction may play a role as the underlying immunopathology in transient hypogammaglobulinemia of infancy (THI) and a selective IgA deficiency (sIgAD) in children $[3,4]$. The skewed immune response may impair tolerance to environmental antigens and result in increased predisposition to allergic diseases and asthma being the most common clinical feature in children with $\mathrm{THI}$, present in as many as $52 \%$ of patients in the study by Keles et al. [5] and in 20\% of patients reported by Ozcan et al. [6]. In patients with a selective and partial IgA deficiency, inadequate mucosal protection due to the deficiency of secretory IgA and facilitated penetration of antigens through the respiratory and gastrointestinal mucosal barrier may contribute to the development of a respiratory and food allergy, the overall incidence of which has been estimated at $88 \%$ of patients in the study by Aghamohammadi et al. [7]. The most common clinical allergic phenotypes associated with sIgAD are rhinoconjunctivitis, asthma, atopic dermatitis and urticaria as well as anaphylaxis, the most severe life-threatening systemic allergic reaction, most frequently being a manifestation of food allergy [8].

\section{Aim}

The aim of the study was to estimate the incidence of food allergy and its clinical manifestation in children with hypogammaglobulinemia. We also aimed to evaluate a correlation between food allergy and various types of antibody production defects in the children studied.

Table 1. Reverence values of major classes of immunoglobulins in different age intervals used in the university hospital central laboratory

\begin{tabular}{lccc}
\hline \multirow{2}{*}{ Age } & \multicolumn{3}{c}{ Reference values [mg/dl] } \\
\cline { 2 - 4 } & IgG & IgA & IgM \\
\hline 1-3 months & $270-780$ & $6-58$ & $12-87$ \\
\hline 4-6 months & $190-860$ & $10-96$ & $25-120$ \\
\hline 7-12 months & $350-1180$ & $36-165$ & $30-104$ \\
\hline 2-3 years & $520-1360$ & $45-135$ & $46-190$ \\
\hline 4-5 years & $540-1420$ & $52-220$ & $40-200$ \\
\hline 6-7 years & $570-1410$ & $65-240$ & $55-210$ \\
\hline 8-9 years & $730-1410$ & $108-200$ & $55-175$ \\
\hline $10-11$ years & $730-1350$ & $91-255$ & $66-155$ \\
\hline $12-13$ years & $770-1510$ & $108-325$ & $70-150$ \\
\hline 14 years to adults & $700-1600$ & $70-400$ & $40-240$ \\
\hline
\end{tabular}

\section{Material and methods}

\section{Patients}

We performed a retrospective review of medical records of 210 children, 113 boys and 97 girls, aged from 2 to 60 months (mean age 17 months), who were hospitalized in the pediatric pneumonology, allergology and immunology clinic due to an acute obstructive airway disease such as bronchiolitis or obstructive bronchitis, between January 2014 and December 2014. All children were assigned to one of two study groups depending upon serum concentrations of major classes of immunoglobulins. Hypogammaglobulinemia concerning one, two or three immunoglobulin isotypes 2 SD below the range of age-matched reference values, according to European Society for Immunodeficiencies (ESID) criteria [9] was evaluated in 78 children and normal levels of immunoglobulins were observed in 132 children studied. The anamnesis and clinical evaluation did not reveal any significant disorders affecting the general health status of any of the participating children, validating the inclusion to one of two groups according to normal or deficient antibody production. The exclusion criteria were the following: prematurity below the 37 week of gestation for the purpose of eliminating the influence of the immunodeficiency of prematurity to gastrointestinal manifestations in the children studied, neurological disorders including cerebral palsy that could be associated with gastrointestinal dysfunctions, and metabolic diseases including cystic fibrosis and congenital anatomical anomalies of the upper and lower airways as well as of the gastrointestinal tract that could mimic the symptoms of food allergy.

\section{Methods}

We performed a detailed analysis of food allergy-related symptoms such as eczema, recurrent episodes of abdominal pain, abnormal stools (loose stools, also with an admixture of blood and/or mucus), constipation, spitting and vomiting following ingestion of the sensitizing food in all children studied. The diagnosis of food allergy in the children studied was based on parent reporting and oral food challenges (OFC). The incidence of the aforementioned symptoms was correlated with concentrations of major classes of immunoglobulins in serum. The levels of serum immunoglobulin isotypes were examined with the use of immunoturbidimetric method (Beckman Coulter, USA). The age-matched reference values of major classes of immunoglobulins in children of different age groups valid in our hospital laboratory are displayed in Table 1. The total serum IgE concentration was measured with the use of ImmunoCap system (Pharmacia Diagnostics, USA). As our analysis revealed, allergen-specific IgE antibodies were examined in only $35(17 \%)$ of all participating children and because of a poor correlation between symptoms presented and 
food-specific IgEs, the results are not displayed in the paper.

\section{Statistical analysis}

For the purpose of assessing the relationships between nominal variables, Pearson's chi-squared $\left(\chi^{2}\right)$ test and the $\chi^{2}$ likelihood ratio were employed. Additionally, for fourfold tables Fisher's exact test was used. P-values $<0.05$ were considered as statistically significant.

\section{Results}

A group of 210 children, 78 children with hypogammaglobulinemia, aged from 2 to 60 months (mean age: 17 months) and 132 children with normal serum immunoglobulin levels, aged from 1 to 54 months (mean age: 10 months), were enrolled in the study. Among all the children studied, there were 117 boys and 93 girls, and the boys were more often affected by the antibody deficiency than the girls ( $n=42$ (54\%) and $n=36$ (46\%), respectively) as shown in the PedPAD Study [10].

Basic anthropometric parameters defining the nutritional status, such as body mass, growth and body mass values, were within a broad normal range, between the $10^{\text {th }}$ and $90^{\text {th }}$ percentiles, in the vast majority of all participating children $(n=153$ (73\%) and 166 (79\%), respectively).

A group of the children with hypogammaglobulinemia consisted of 25 (32\%) children with a combined IgG and IgA deficiency, 18 (23\%) children were equally affected by an isolated IgA or isolated IgG deficiency and 7 (9\%) children manifested a deficiency in all three major immunoglobulin isotypes.

Altogether, symptoms of cow's milk protein allergy (CMPA) occurred among the hypogammaglobulinemic children in as many as 40 (51\%) of them, compared to 63 (48\%) children with CMPA and normal serum immuno- globulin levels. Although CMPA was observed in hypogammaglobulinemic children with deficiencies of all immunoglobulin isotypes, there was only a statistically significant correlation between CMPA and a selective IgA deficiency which was demonstrated by 4 children $(p<0.05)$.

Isolated CMPA occurred in 29 (37\%) hypogammaglobulinemic children and 10 (13\%) children manifested a concomitant allergy to other alimentary antigens such as hen's egg protein, soy, codfish, wheat, walnut and apple. There was no statistically significant correlation between the number of sensitizing allergens and deficiency of any immunoglobulin isotypes.

Clinical manifestation of food allergy in the children studied included both immediate reactions, with the onset of symptoms within up to 30 min after food ingestion, as well as late-onset reactions, occurring within hours or days after food ingestion. In children with antibody deficiencies, immediate clinical reactions such as vomiting and intensive spitting occurred in 11 (14\%) of them. Late-onset reactions, which were definitely more frequent and predominating in the symptomatology, included atopic dermatitis (observable in 46 (59\%) hypogammaglobulinemic children), infantile abdominal colic, chronic diarrhea and poor weight gain as shown in Table 1. The tendency for the skin involvement was more frequent than gastrointestinal symptoms, which were observable in 32 (41\%) patients. Atopic dermatitis was a major manifestation of food allergy in all four of the most common antibody deficiencies in the children studied, namely in a combined IgG and IgA deficiency, isolated IgG and isolated IgA deficiencies, as well as in a combined deficiency of three major immunoglobulin isotypes - see Table 2. It is worth noting that there was a statistically significant correlation between atopic dermatitis as a chief manifestation of food allergy and an isolated IgA deficiency $(p<0.05)$. In contrast to antibody deficient children, patients in the control group with

Table 2. The incidence of immediate-onset and late-onset allergic reactions to foods in hypogammaglobulinemic and normogammaglobulinemic children

\begin{tabular}{|c|c|c|}
\hline \multicolumn{3}{|c|}{ Types of allergic reactions to foods } \\
\hline Immunoglobulin production & $\begin{array}{l}\text { Immediate-onset reactions } \\
\text { (incidence) }\end{array}$ & $\begin{array}{l}\text { Late-onset reactions } \\
\text { (incidence) }\end{array}$ \\
\hline Children with hypogammaglobulinemia & $\begin{array}{l}\text { Gastrointestinal (14\%): } \\
\text { - vomiting } \\
\text { - intensive spitting }\end{array}$ & $\begin{array}{l}\text { Gastrointestinal ( } 41 \% \text { in total): } \\
\text { - infantile abdominal colic }(36 \%) \\
\text { - chronic diarrhea }(28 \%) \\
\text { - poor weight gain }(6 \%) \\
\text { - atopic dermatitis }(59 \%)\end{array}$ \\
\hline $\begin{array}{l}\text { Children with } \\
\text { normogammaglobulinemia }\end{array}$ & $\begin{array}{l}\text { Gastrointestinal (20\%): } \\
\text { - vomiting } \\
\text { - intensive spitting }\end{array}$ & $\begin{array}{l}\text { Gastrointestinal ( } 65 \% \text { in total): } \\
\text { - infantile abdominal colic (32\%) } \\
\text { - chronic diarrhea ( } 24 \%) \\
\text { - flatulence }(10 \%) \\
\text { - constipation }(10 \%) \\
\text { - poor weight gain }(5 \%) \\
\text { - atopic dermatitis }(54 \%)\end{array}$ \\
\hline
\end{tabular}


Table 3. The incidence of gastrointestinal symptoms and atopic dermatitis in children with different types of hypogammaglobulinemias

\begin{tabular}{lcccccc}
\hline \multirow{2}{*}{$\begin{array}{l}\text { Clinical } \\
\text { manifestations } \\
\text { of food allergy }\end{array}$} & IgG deficit & IgA deficit & IgM deficit & IgG + IgA deficit & IgG + IgM deficit & IgG + IgA + IgM deficit \\
\cline { 2 - 6 } & $8(10 \%)$ & $4(5 \%)$ & $1(1 \%)$ & $10(13 \%)$ & $1(1 \%)$ & $4(5 \%)$ \\
\hline $\begin{array}{l}\text { Atopic dermatitis } \\
\begin{array}{l}\text { Gastrointestinal } \\
\text { disorders }\end{array}\end{array}$ & $4(5 \%)$ & $0(0 \%)$ & $0(0 \%)$ & $5(6 \%)$ & $0(0 \%)$ & $3(4 \%)$ \\
\hline
\end{tabular}

normal immunoglobulin levels presented symptoms of gastrointestinal reactions more frequently than atopic dermatitis (86 (65\%) children vs. 71 (54\%) children). As in the case of hypogammaglobulinemic children, in the control group, late-onset symptoms were observable most frequently, with infantile abdominal colic, chronic diarrhea, flatulence, constipation and poor weight gain (data summarized in Table 3).

The incidence of serum total lgE levels which were elevated above the age-matched reference values was observed in 7 (9\%) children with hypogammaglobulinemia and in 20 (15\%) children with normal immunoglobulin production as shown in Figure 1.

\section{Discussion}

The increasing problem of food allergy in children poses important questions about the pathogenesis of this disease and the role of likely environmental conditions, as well as intrinsic predisposing factors. Among the mechanisms proposed, early life exposures associated with modern urban development leading to a breakdown of oral tolerance are particularly underscored. Declining biodiversity, indoor lifestyles, and dietary patterns in combination with numerous genetic and epigenetic factors modulate the immune response to food allergens [11]. Along with the environmental risk factors, the host's immune system with its both innate and adaptive arms ensures the integrity of the skin and mucous membrane barriers and serves as a protection against harmful external antigens. Therefore, defects in mounting a sufficient response against penetrating pathogens and failure to support the commensal organisms in the gastrointestinal tract in immunodeficient children predispose them to the breakdown of oral tolerance and to the subsequent development of food allergy, inflammatory bowel disease and infections [11]. Furthermore, the gastrointestinal tract is the largest immunological organ in the human body, rich in antibody-secreting plasma cells

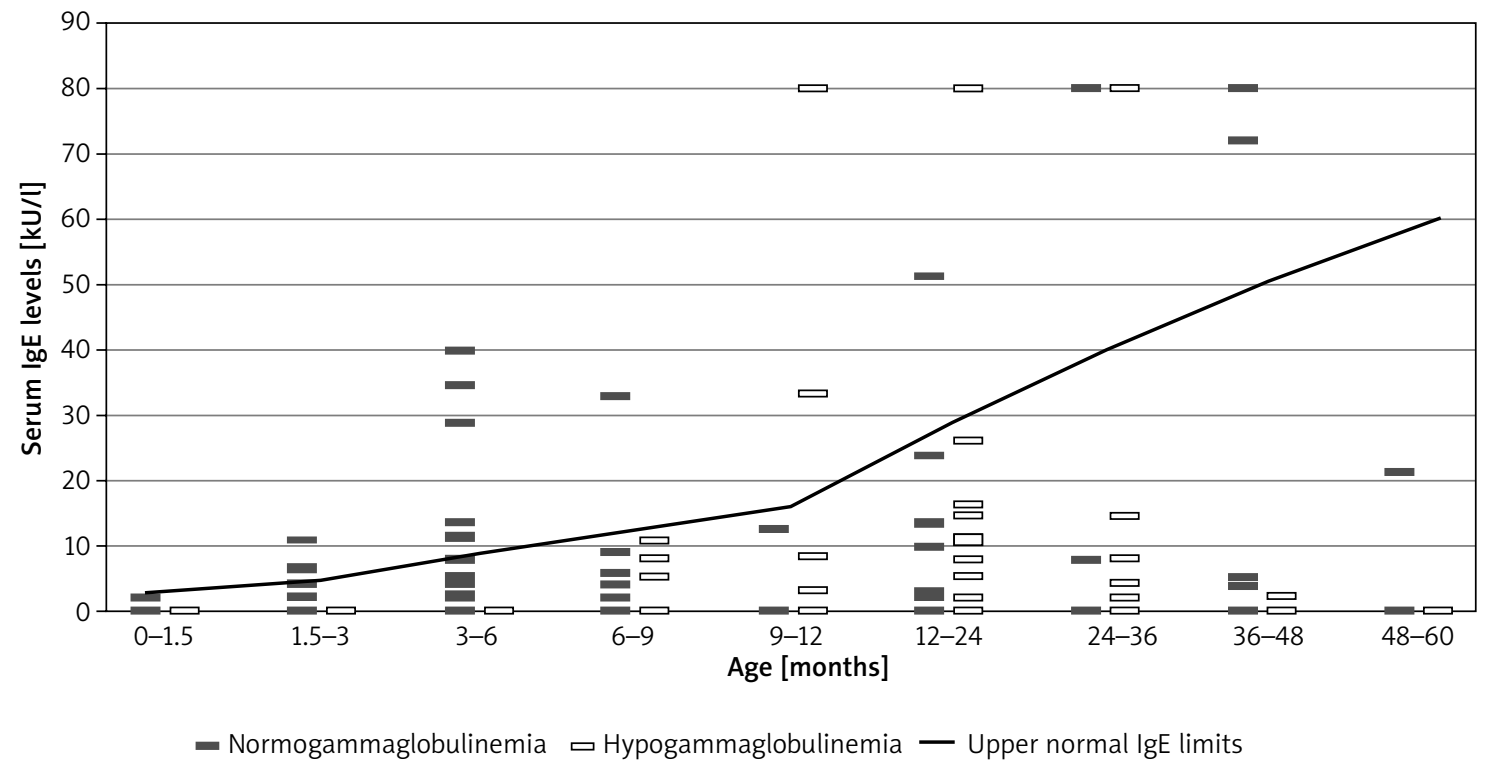

Figure 1. IgE levels in children with hypogammaglobulinemia vs. in children with normogammaglobulinemia in different age groups 
and an essential source of mucosal and circulating IgA and, to a lesser extent, also IgM and IgG isotypes playing a prominent role in mucosal compartmentalization of food antigens and protecting against allergic sensitization to foods [12-14]. Systemic sensitization to foods may thus reflect a lack of appropriate, antibody-dependent compartmentalization of food antigens. As mucosal secretory immunoglobulin levels are not determined, children with hypogammaglobulinemia, particularly IgA deficient ones may not provide sufficient local protective functions in the gastrointestinal tract. Indeed, a selective IgA deficiency in children has been proved to be associated with the increased risk of allergy to foods in several clinical studies from Europe $[15,16]$, the Middle East [7] and in the report of the United States Immunodeficiency Network which was published recently [17].

Interestingly, in the hypogammaglobulinemic children studied, atopic dermatitis was the most prominent manifestation of allergy to foods, present in $59 \%$ of them. In our stud, all IgA-deficient children manifested cutaneous symptoms of food allergy and while atopic dermatitis occurred in patients with all kinds of hypogammaglobulinemia, solely in IgA-deficient children studied; the correlation between symptoms and immunodeficiency was statistically significant. This finding is consistent with observations also made by other authors who reported an association of impaired antibody production with atopic dermatitis in children $[6,18]$, and which was also found in our previous study [19]. Interestingly, it has been shown recently that cutaneous sensitization may predispose patients to an intestinal allergy by the increased production of thymic stromal lymphopoietin (TSLP) and subsequent T-helper type 2 (Th2)-dependent immune responses to food antigens in the skin $[20,21]$. These studies may be helpful in understanding the mechanisms by which atopic dermatitis progresses to allergic immune responses at other mucosal surfaces [11] and shedding light on the role of atopic dermatitis in development of food allergy in hypogammaglobulinemic children predisposed to damage at the large surface area of the skin and mucous membrane of the gastrointestinal tract.

An emerging problem in children with antibody production defects and symptoms of food allergy is an appropriate diagnostic approach concerning serum immunoglobulin E values, the first-line test in assessing IgE-mediated sensitization. In our study, an atopic background was proven in only $9 \%$ of children with hypogammaglobulinemia and in $15 \%$ of children with normal immunoglobulin production. These data may suggest a growing importance and high incidence of non-lgE-mediated food allergic disorders in the children studied, accounting for up to $40 \%$ of milk protein allergy in infants and young children [22,23] and which manifest as profuse enterocolitis syndrome with diarrhea, repetitive vomiting and failure to thrive or as benign allergic proctocolitis with bloody stools. However, in children with hypogammaglobulinemia, as to isolated or combined immunoglobulin deficiencies, low serum IgE may rather reflect a multiple immunoglobulin deficit and therefore, the role of the non-lgE mediated allergic pathway in this group of immunodeficient children must be particularly emphasized [24, 25].

\section{Conclusions}

Allergic sensitization to foods is a frequent problem in infants and young children and this phenomenon affects both antibody-deficient patients as well as patients with normal immunoglobulin production. Although the incidence of food allergy is comparable in the children studied and in the control group, IgA deficiency poses an increased risk of developmental allergic reactions to foods, manifesting as intestinal symptoms or atopic dermatitis. Furthermore, the pattern of allergic sensitization is remarkably variant with non-lgE mediated gastrointestinal disorders and cutaneous manifestations. Thus, children with a selective IgA deficiency should be evaluated for food allergy if recurrent or persistent gastrointestinal symptoms or eczema are observable.

\section{Conflict of interest}

The authors declare no conflict of interest.

\section{References}

1. De Vries E, Driessen G. Educational paper: Primary immunodeficiencies in children: a diagnostic challenge. Eur J Pediatr 2011; 170: 169-77.

2. Driessen G, van der Burg M. Educational paper: Primary antibody deficiencies. Eur J Pediatr 2011; 170: 693-702.

3. Kowalczyk D, Baran J, Webster AD, Zembala M. Intracellular cytokine production by Th1/Th2 lymphocytes and monocytes of children with symptomatic transient hypogammaglobulinemia of infancy (THI) and selective IgA deficiency (SIgAD). Clin Exp Immunol 2002; 127: 507-12.

4. Rutkowska M, Trzyna E, Lenart M, et al. The levated numer of circulating regulatory $T$ cell in patients with transient hypogammaglobulinemia of infancy is not associated with any abnormalities in the genes encoding the TGF-beta receptors. Clin Immunol 2013; 149: 83-5.

5. Keles S, Artac H, Kara R, et al. Transient hypogammaglobulinemia and unclassified hypogammaglobulinemia: similarities and differences. Pediatr Allergy Immunol 2010; 21: 843-51.

6. Ozcan C, Metin A, Ercoglu M, Kocabas CN. Allergic diseases in children with primary immunodeficiencies. Turk J Pediatr 2014; 56: 41-7.

7. Aghamohammadi A, Cheraghi T, Gharagozlou M, et al. IgA deficiency: correlation between clinical and immunological phenotypes. J Clin Immunol 2009; 29: 130-6.

8. Story RE. Manifestations of food allergy in infants and children. Pediatr Ann 2008; 37: 530-5.

9. De Vries E; European Society for Immunodeficiencies (ESID) members. Patient-centred screening for primary immunodeficiency, a multi-stage diagnostic protocol designed for non-immunologists: 2011 update. Clin Exp Immunol 2012; 167: 108-19. 
10. Schatorje EJ, Gathmann B, van Hout RW, de Vries E; PedPAD consortium. The PedPAD study: boys predominate in the hypogammaglobulinemia registry of the ESID online. Clin Exp Immunol 2014; 176: 387-93.

11. Ashley S, Dang T, Koplin J, et al. Food for thought: progress in understanding the causes and mechanisms of food allergy. Curr Opin Allergy Clin Immunol 2015; 15: 237-42.

12. Agarwal S, Mayer L. Diagnosis and treatment of gastrointestinal disorders in patients with primary immunodeficiency. Clin Gastroenterol Hepatol 2013; 11: 1050-63.

13. Berin C. Mucosal antibodies in the regulation of tolerance and allergy to foods. Semin Immunopathol 2012; 34: 633-42.

14. Corthesy B. Multi-faceted functions of secretory IgA at mucosal surfaces. Front Immunol 2013; 4: 185.

15. Nurkic J, Numanovic F, Arnautalic L, et al. Diagnostic significance of reduced IgA in children. Med Arch 2014; 68: 381-3.

16. Janzi M, Kull I, Sjoberg R, et al. Selective IgA deficiency in early life: association to infections and allergic diseases during childhood. Clin Immunol 2009; 133: 78-85.

17. Tuano K, Orange JS, Sullivan K, et al. Food allergy in patients with primary immunodeficiency diseases: Prevalence within the US Immunodeficiency Network (USIDNET). J Allergy Clin Immunol 2015; 135: 273-5.

18. Aytekin C, Tuygun N, Gokce S, et al. Selective IgA deficiency: clinical and laboratory features of 118 children in Turkey. J Clin Immunol 2012; 32: 961-6.

19. Szczawińska-Popłonyk A, Bręborowicz A, Ossowska L. Food allergy in children with hypogammaglobulinemia. Pediatr Pol 2012; 87: 444-8.

20. Noti M, Kim BS, Siracusa MC, et al. Exposure to food allergens through inflamed skin promotes intestinal food allergy through the thymic stromal lymphopoietin-based axis. J Allergy Clin Immunol 2014; 133: 1390-9.

21. Han H, Thelen TD, Comeau MR, Ziegler SF. Thymic stromal lymphopoietin-mediated epicutaneous inflammation promotes acute diarrhea and anaphylaxis. J Clin Invest 2014; 124: 5442-52.

22. Nowak-Węgrzyn A. Food protein-induced enterocolitis syndrome and allergic proctocolitis. Allergy Asthma Proc 2015; 36: $172-84$

23. Comberiati P, Cipriani F, Schwarz A, et al. Diagnosis and treatment of pediatric food allergy: an update. Ital J Pediatr 2015; 41: 13.

24. McVicker S, Karim MY. IgE deficiency may indicate underlying hypogammaglobulinaemia? I Clin Pathol 2014; 67: 832-3.

25. Unsworth DJ, Virgo PF, Lock RJ. Immunoglobulin E deficiency: a forgotten clue pointing to possible immunodeficiency? Ann Clin Biochem 2011; 48: 459-61. 